\title{
SIMULATION STUDY OF A THERMIONIC RF GUN FOR HIGH BRIGHTNESS AND SHORT PULSE BEAM
}

\author{
T. Tanaka ${ }^{\#}$, F. Hinode, M. Kawai, A. Miyamoto ${ }^{*}$, K. Shinto ${ }^{* *}$, H. Hama, \\ Laboratory of Nuclear Science, Tohoku University, \\ 1-2-1 Mikamine, Taihaku-ku, Sendai 982-0826, Japan
}

\begin{abstract}
Recently sub-picosecond electron pulses are paid attention because of valious applications of $\mathrm{THz}$ coherent radiation emitted from such short bunches. Although photoinjetcors using RF guns are rapidly developped as high blliiant electron sources, while thermionic RF guns are still expected to have potential ability to create highbrightness and short-pulse beams [1]. In particular, components of a thermionic RF gun are simple, compact and low-cost than those of a photo-cathode RF gun. For creating such beams, a prototype of thermionic RF gun was designed and its characteristics have been studied by a 3-D simulation code based on an FDTD (Finite Difference Time Demain) method which has been developed so far [2]. The gun is consists of two independently power feeding S-band RF cavities. The first cell is a cathode cell to extract the beam and the second one is an accelerating cell. This gun can be operated at modes with different RF-power ratio and phase between two RFs. A similar way of operating RF gun has been already reported by Lewellen [3]. This paper describes the results of simulations for this thermionic RF gun.
\end{abstract}

\section{INTRODUCTION}

Conventional way to compress the bunch length from the thermionic RF gun is employing an $\alpha$-magnet [4]. Though the $\alpha$-magnet is apparently working skilful manipulation of longitudinal phase space, it seems to be very sensitive to the tolerance of the magnet alignment and the accuracy of the magnetic field.

Beside the problem of backbombardment [5], there is a specific feature in the longitudinal dynamics in thermionic RF guns. At the beginning of the beam extraction, the head of the electrons from cathode is followed immediately by the electrons just behind, which is extracted by the higher electric field than that at the head of the beam train. Thus later electrons would get kinetic momentum or velocity faster than the head of the electrons. Consequently the electrons are expected to concentrates on the head of the beam under certain conditions such as the gun geometry and the strength of the RF field. This velocity-bunching-like phenomenon has already known by developers of the thermionic RF guns [1]. According to our simulation study so far, this

\footnotetext{
takumi@Ins.tohoku.ac.jp

Present address: ${ }^{*}$ Hiroshima Synchrotron Radiation Center, Hiroshima University, 2-313 Kagamiyama, Higashi-Hiroshima 739-0046, Japan

** Department of Quantum Science and Energy Engineering, Tohoku

University, 6-6 Aramaki Aza Aoba, Aoba-ku, Sendai 980-8579, Japan
}

velocity-bunched sharp peak on the head of the beam train might possibly reach to a pulse width of $\sim 200 \mathrm{fs}$ including the electrons of a couple of tens $\mathrm{pC}$.

If the velocity-bunching-like effect, which occurs at the beginning of the rising up of the longitudinal electric field, will be able to be enhanced, the sub-picosecond beam might be obtained without any bunch compressors. In general, the optimization of the (multi-cell) RF gun resulted from careful simulation of the motion of the electrons and calculation of the RF fields to determine the shape of the cavities. However in this case remaining parameters that can be varied is only the input RF power.

We have considered an RF gun of a very low coupling constant between two cells, so that the electric fields and phase between cells are independently varied to find optimum parameters for both the best velocity-bunching and the transverse quality of the beam.

Table 1: Target parameters of the thermionic RF gun

\begin{tabular}{lc}
\hline RF freqency & $2,856 \mathrm{MHz}$ (S-band) \\
Energy @ exit of gun & $\sim 2 \mathrm{MeV}$ \\
Velocity: $\beta=v / c$ & $0.968 @ 2 \mathrm{MeV}$ \\
Number of cells & 2 \\
Bunch length (rms) & several hundred fs \\
Bunch charge & $\sim 0.1 \mathrm{nC}$ \\
Macropulse current & $\sim 280 \mathrm{~mA}$ \\
$\varepsilon_{\text {norm. rms }}$ & $<10 \pi \mathrm{mm} \mathrm{mrad}^{\text {Material of cathode }} \mathrm{LaB}_{6}$ \\
Cathode radius & $-2 \mathrm{~mm}$ \\
\hline
\end{tabular}

\section{CHARACTERISTICS OF THE ITC-RF GUN}

The ITC (Independently Tunable Cells) RF Gun has been designed so as to vary the relative phase and the amplitude of RF fields in an accelerating cell (2nd cell), because a timing of which the head of the extracted bunch train gets into the next accelerating filed would be very significant for the velocity-bunching.

We performed a basic design of the ITC-RF gun by using a code SUPERFISH. Because kinetic energy gain in the 1 st cell was reduced to be less than $\sim 0.5 \mathrm{MeV}$ to enhance the velocity-bunching, the length of the 1st cell (from the cathode to the end of 1st cell) should be not too long. In addition, in order to obtain almost no RF coupling between two cells, a radius of the center beam duct has been chosen to be $4 \mathrm{~mm}$.

Results of the calculation of the RF field for each cell using SUPERFISH are shown in Fig. 1. 

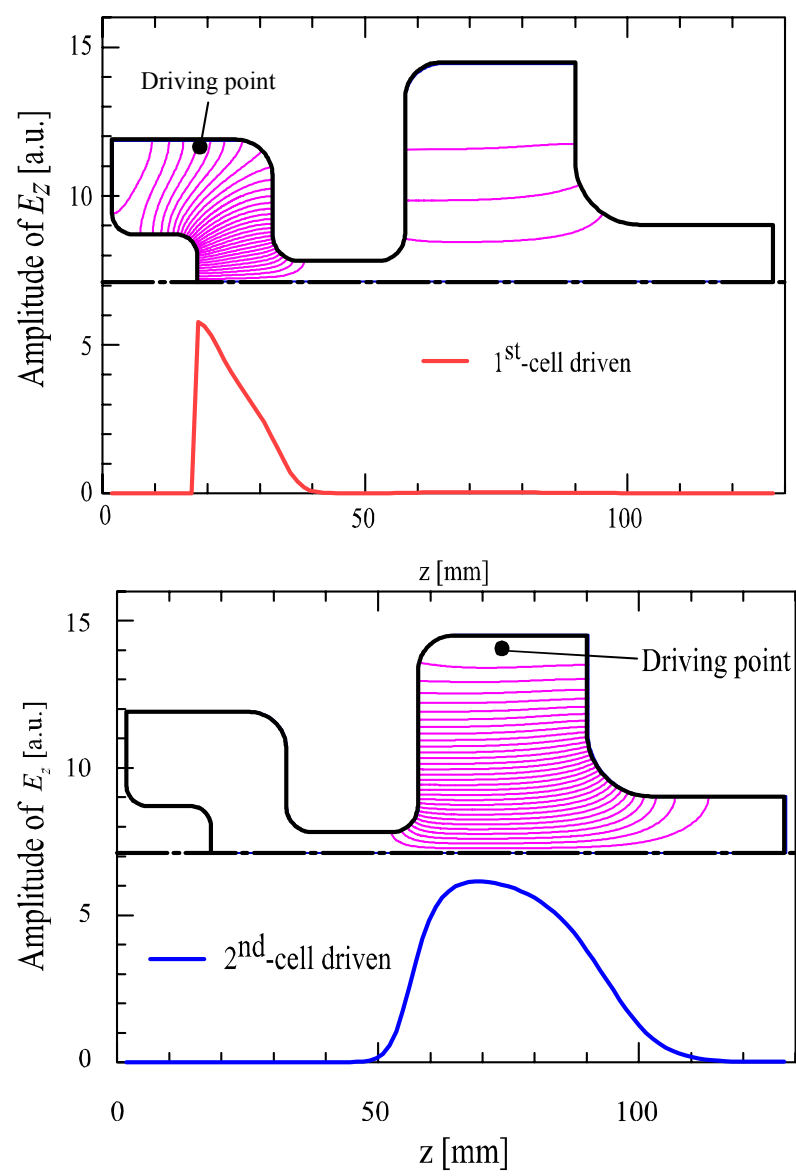

Figure 1: Calculated electric field lines and longitudinal electric field strengths along the beam axis are shown. Upper figure shows a case the only 1 st cell is excited, and lower one for the 2 nd cell.

\section{FDTD SIMULATION}

\section{FDTD 3-D simulation}

A code of Maxwell's equations solver using an FDTD method has been developed to simulate propagation of the microwave for a boundary condition of the RF gun. The equation of motion of the macroparticle is simultaneously solved in the code so that the effects originated from the charged particles current (i.e., space charge) are automatically included in Maxwell's equations. The detail of the code can be found in ref. [2]. One of problems in this method, the accuracy is not better than 2$\mathrm{D}$ simulations because of finite memories of the PCs. The shape of the gun consisted of cubic-like grids (or cells) is not completely reproduced. However the code shows the beam dynamics in the gun including space charge and the beam loading effects, which is very important information for designing RF devices in accelerators.

\section{Results of the simulation}

A snap shot of the beam acceleration in the gun shows in Fig. 2. The head of the extracted beam train is just passing through the center of the iris. The phase difference between two cells, here the simulation, is almost $\pi$-mode like. We firstly examined dependence on the field strength.

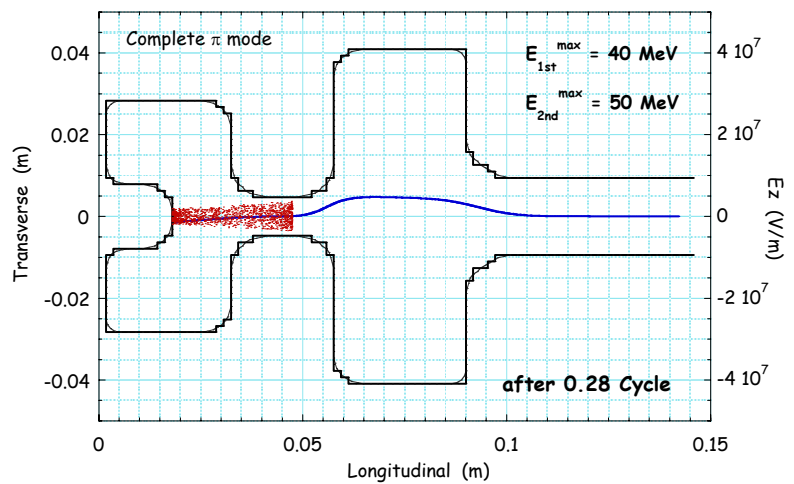

Figure 2: A snap shot of the simulation. The time is counted from when the longitudinal electric field on the axis is 0 .

In order to clarify the velocity-bunching in the ITC-RF gun, the particle distributions along the axis during the acceleration are shown in Fig. 3.

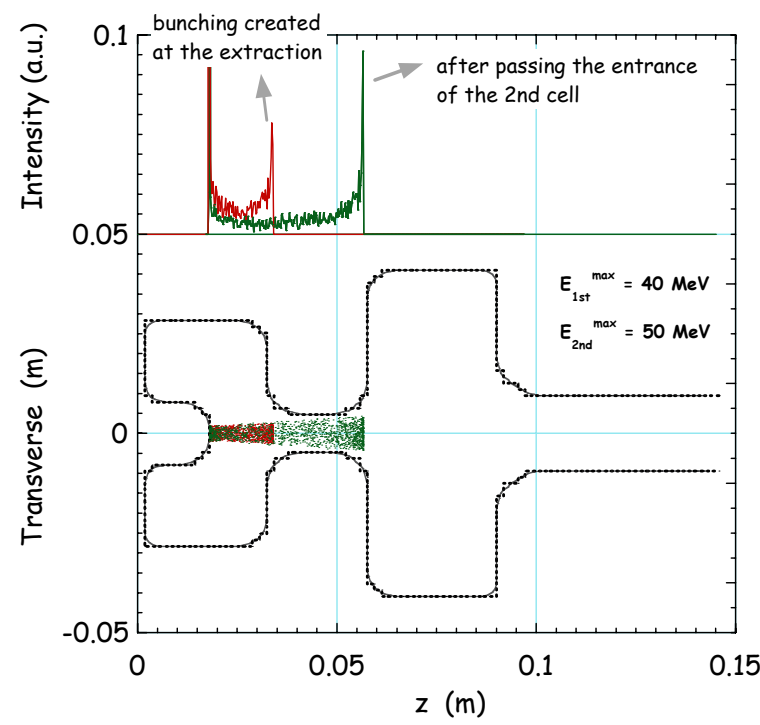

Figure 3: The peak around $\mathrm{z} \sim 3 \mathrm{~cm}$ is created when the beam extracted from the cathode at $\mathrm{t} \sim 0$. Another sharp peak is enhanced when the head of the beam train is getting into the 2nd cell field. Particle locations in the gun corresponding to upper figure(lower).

Degree of the velocity-bunching is of course strongly depending on the RF field strength and the relative phase. Other beam properties, such as energies, transverse emittances and beam currents are also governed by the parameters of the RF fields. In this report, we have just investigated a dependence on the filed strength of the 1 st cell, so that the phase is fixed to be $\pi$-mode and the maximum field strength of 2 nd cell is kept at $50 \mathrm{MV} / \mathrm{m}$. 


\section{LONGITUDINAL PHASE SPACE}

Figure 4 shows the final longitudinal phase spaces at the exit of the ITC-RF gun for various RF strengths of the extraction cell.

Since the final energy of the extracted beam from the thermionic RF gun is continuum spectrum, the longitudinal phase spaces for regions of only $4 \%$ from the top kinetic energies are shown. As one can see, the $\mathrm{RF}$ strength of more than $\sim 50 \mathrm{MV} / \mathrm{m}$, the maximum energy is not at the head of the bunch train. Such phase spaces are not suitable to select the beam energy and the pulse duration.

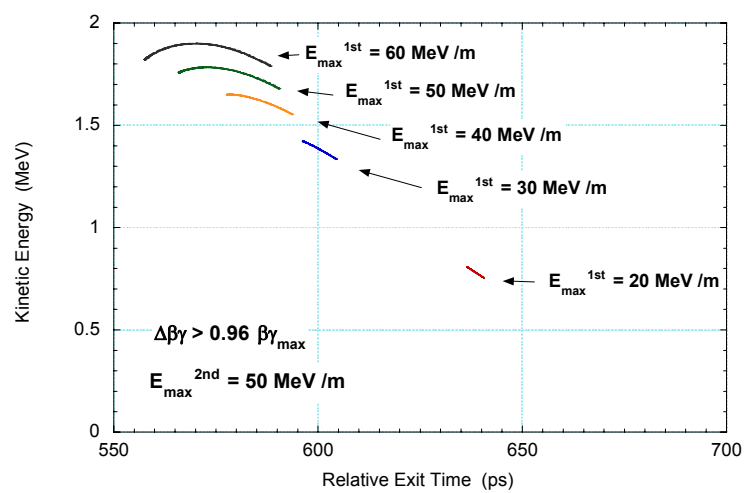

Figure 4: Longitudinal phase spaces at the exit of the gun. Field strengths of the 1st cell are denoted in the figure. The phase relative to 2 nd cell is fixed to be $\pi$, and the maximum strength of 2 nd cell is $50 \mathrm{MV} / \mathrm{m}$.

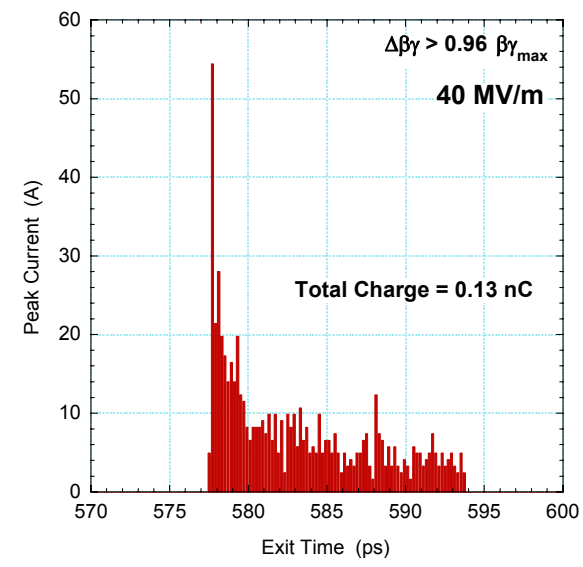

Figure 5: Time distribution of the beam bunch extracted by $40 \mathrm{MV} / \mathrm{m}$ and $50 \mathrm{MV} / \mathrm{m}$ maximum RF fields in the $1 \mathrm{st}$ cell and the 2 nd cell, respectively. The time bin in the figure is $0.2 \mathrm{ps}$.

The number of electrons in the region of $\Delta \beta \gamma>0.96$ $\beta \gamma_{\max }$ is monotonically increased with the extraction voltage. Total charge of $0.1 \mathrm{nC}$ is obtained when the extraction filed is $35 \mathrm{MV} / \mathrm{m}$ (assuming an emission current of the cathode is $100 \mathrm{~A} / \mathrm{cm}^{2}$ so far). If we use $\mathrm{LaB}_{6}$ as a cathode material, we might expect to obtain two or three times more.
A projected spectrum of the phase space onto the time axis at the $40 \mathrm{MV} / \mathrm{m}$ extraction field is shown in Figure 5. The electrons are concentrated into the head of the bunch is well seen. Because number of particle statistics is poor in this simulation, it seems to be difficult to know an accurate distribution of the bunch.

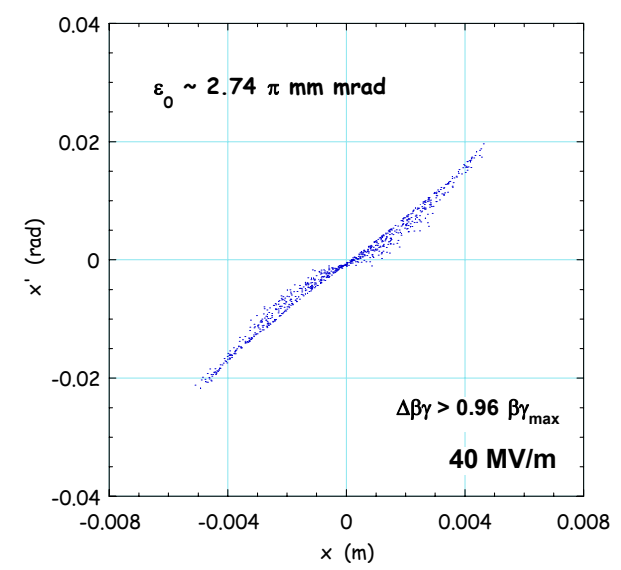

Figure 6: Transverse phase space of the identical bunch shown in Fig. 5. The normalized emittance is approximately $3 \pi \mathrm{mm} \mathrm{mrad}$,

\section{SUMMARY}

A time duration of $1 \mathrm{ps}$ from the head of the bunch contains only $30 \mathrm{pC}$ and the FWHM (if we cut the tail part) might be $\sim 300 \mathrm{fs}$. These numbers, particularly the total charge, do not satisfy our future project. However we have not applied appropriate optimization for the ITC$\mathrm{RF}$ gun. In addition another variable parameter, the phase, is not employed yet in the simulation. At the moment, we have thought there will be enough room to much improve the performance of the ITC-RF gun. The transverse emittance is also not better in spite of lower charge, which is due to a stronger electric field of the radial direction in the 1 st cell because of the preliminary design.

Further design work and the simulation are under way.

\section{REFERENCES}

[1] P. Kung, et al., Phys. Rev. Lett. 73 (1994) 967;

S. Rimjaem, et al., Nucl. Instr. and Meth. A 533 (2004) 258.

[2] H. Hama, et at., Nucl. Instr. and Meth. A 528 (2004) 371.

[3] J.W. Lewellen, Proc. of the 2003 Particle Accelerator Conf., Portland, Oregon U.S.A., pp. 2035.

[4] H.A. Enge, Rev. Sci. Instrum. 34 (1963) 385;

M. Borland, "A High-brightness Thermionic Microwave Electron Gun,” Ph.D. Thesis, Stanford University, 1991.

[5] C.B. McKee and J.M.J. Madey, Nucl. Instr. and Meth. A 296 (1990) 716. 